Kong. Res. J. 2(1) : 111-114, 2015

ISSN 2349-2694

Kongunadu Arts and Science College, Coimbatore.

\title{
PARTIALLY PURIFIED EXTRACT OF RICINUS COMMUNIS AGAINST DEVELOPMENTAL STAGES OF AEDES AEGYPTI (CULICIDAE: DIPTERA)
}

\author{
Rajmohan, D*, K. Logankumar, V. Sabitha, D. Saranya and T. Sujila \\ PG and Research Department of Zoology, Kongunadu Arts and Science College, Coimbatore-641 029. \\ *E-mail: rajmohandevadass@gmail.com
}

\begin{abstract}
The present study has been taken to study the insecticidal properties of botanical,Ricinus communis on the growth and development of the Chikungunya vector mosquito, Aedes aegypti. Mortality value of egg, larvae and pupae treated with different concentration of the partially purifiedseed extract of Ricinus communis was observed at the end of 24 hours of exposure. The results revealed that the plant, Ricinus communisshowed better response against the developmental stages on the mosquito. All of these studies of the effect of plant extracts on the mosquito susceptibility showed that Ricinus communis may cause death by acting as neurotoxicants on respiratory toxicants by inhibiting the flow of nerve impulse and decrease in oxygen uptake ultimately resulting in death. The preliminary phytochemical analysis shows the presence of certain secondary metabolites like alkaloids, flavonoids in the extracts of experimental plant,Ricinus communis. As the alkaloids, flavonoids are known to have effective mosquitocidal properties. It indicates that the extract ofRicinus communisis most reliable and effective in terms of mosquitocidal properties.
\end{abstract}

Keywords: Ricinus communis, Aedes aegypti, mosquito control, LC50.

\section{INTRODUCTION}

Vector control is of serious concern in developing countries like India. Due to lack of awareness, development of resistance and socio economic reasons, every year a large part of the population is affected by one or more vector borne diseases. Aedes aegypti is a tropical mosquito. It is believed that Aedes aegypti originated from Central Africa, where it is found in greatest abundance. Being a domestic breeder, it found breeding places on sailing ships on those days, where it has been distributed to all parts of the world.

The mosquitoes not only annoy us by their noise and painful bites but also transmit human diseases such as malaria, yellow fever, filariasis, encephalitis etc. In the present study much attention has been focused on the Aedes aegypti since it plays a major role in the transmission of nocturnal periodic form of Chikungunya all over the world. Mosquitoes transmitdiseases like malaria, filariasis,dengue fever and Japanese encephalitis are among the most serious vector- born diseases in developing countries.

The castor oil plant (Ricinus communis) is a species of flowering plant in the spurge family, Euphorbiaceae. It belongs to a monotypicgenus, Ricinus, and subtribe, Ricininae. The evolution of castor and its relation to other species are currently being studied using modern genetic tools.

Its seed is the castor bean, which, despite its name, is not a true bean. Castor is indigenous to the southeastern Mediterranean Basin, Eastern Africa, and India, but is widespread throughout tropical regions (and widely grown elsewhere as an ornamental plant Castor seed is the source of castor oil, which has a wide variety of uses. The seeds contain between $40 \%$ and $60 \%$ oil that is rich in triglycerides, mainly ricinolein. They also contain ricin, a poison, which is also present in lower concentrations throughout the plant. The toxicity of raw castor beans is well-known, and reports of actual poisoning are relatively rare. Children could conceivably die from as few as three beans; adults may require eight or more. As an example of the rarity of castor bean poisoning, in recent years there have only been two cases reported in all of England, and in both the victims recovered uneventfully.

The approach to combat these diseases largely relies on interruption of the disease transmission cycle by either targeting the mosquito larvae at breeding sites through spraying of stagnant water or by killing or repelling the adult mosquitoes (Corbel et al., 2004; Joseph et al., 2004).

Phytochemicals are botanicals which are naturally occurring insecticides obtained from floral resources. Applications of phytochemicals in 
mosquito control were in use since the 1920s (Shahi, et al., 2010). The extract of this plant was administrated on egg and larval stages because the best option for mosquito control is to target these aquatic stages rather than the adults

\section{MATERIALS AND METHODS}

\subsection{Selection of mosquito species}

An important vector species of mosquito Aedes aegypti is selected for the presented study. Aegypti is the principle vector of chikungunya, dengue fever and dengue hemorrhagic fever and it is reported to infect more than hundred million people every year and more than 110 countries in the tropics (Halstead, 2000).

\subsection{Mosquito rearing}

Mosquito colony maintained at $28 \pm 2^{\circ} \mathrm{C}, 70 \pm$ $10 \%$ Relative humidity and a photoperiod of $12: 12$, L: D at the Zoology Research Department, Kongunadu Arts and Science College, Coimbatore.

\subsection{Mosquito feeding}

Larval forms are maintained in trays by providing dog biscuits and yeast powder in the $3: 1$ ratio. Adult are provided with $10 \%$ sucrose solution and one week old chick for blood meal.

\subsection{Selection of the plants}

The experimental plant was collected from The Nilgiri district, Tamilnadu, India. The experimental plant is Ricinus communis

\section{plant}

\subsection{Preparation of seed extract of experimental}

The experimental plant was collected from the Nilgiri district and brought to the laboratory. The separated seeds were dried under shade at room temperature $\left(28 \pm 1^{\circ} \mathrm{C}\right)$ for about 20 days. The completely dried seeds were powdered and sieved to get fine powder of seed. The seed extract were obtained by using Soxhlet apparatus.

Two hundred fifty grams of seed powder were dissolved in $200 \mathrm{ml}$ of solvent separately and extract in the Soxhlet apparatus for 8 hours over a mantle heater at $55^{\circ} \mathrm{C}$. The extracts were concentrated using a vacuum evaporator at $45^{\circ} \mathrm{C}$ under low pressure. After complete evaporation of the solvent, the concentrated extract was collected and stored in a refrigerator for later use.

\subsection{Preparation of stock solution and different concentration of seed extract}

One gram of the concentrated extract of dried seeds of experimental plant wasdissolved in $100 \mathrm{ml}$ of water separately and kept as stock solutions. These stock solutions were used to prepare the desiredconcentrations of the extract for exposure of the mosquito egg, larvae and pupae.

\subsection{Partial purification of plant extracts}

Different parts of the plants were taken based on the effect of crude extracts tested to purify on silica gel column. Sufficient quantity of powdered plant materials were dissolved in $60 \%$ acetone and extracted for 8 hrs. Clear supernatant was air dried concentrated and dissolved in acetone. Column was packed with silica gel (60x120 mesh) and washed with $1 \%$ acetone several times. Sample was centrifuged at $5000 \mathrm{rpm}$ for 2 minutes. The clear supernatant was applied over the column eluted with $1 \%$ acetone. Fraction collected $3 \mathrm{ml}$ per minute and were air dried and used for bioassay

To obtain the different concentration of test medium for crude 1 to $10 \mathrm{gm}$ of stock powder and foe silica gel fractions $1 \mathrm{mg}$ of dried powder was dispersed in $100 \mathrm{ml}$ of $0.02 \%$ acetone. The effect of crude and silica gel fractions on the development was noticed for a period of $24 \mathrm{hrs}$.

\subsection{Treatment of egg, larvae and pupae with different concentration of the experimental plants extracts}

In the present study, for treatment of egg, larvae and pupae with the extracts of different experimental plants, $100 \mathrm{ml}$ of tap water was kept in a series of glass beakers (200 $\mathrm{ml}$ of capacity). Required quantity of stock solution (containing 10 $\mathrm{mg} / \mathrm{ml}$ ) was added into each beaker (containing 100 $\mathrm{ml}$ of tap water) to obtain a particular concentration of the extract

Control medium was also maintained with $100 \mathrm{ml}$ of tap water added with the maximum quantity of solvent present in the stock solution of the extract. Separate series of exposure medium with desired concentration of extracts were kept for Aedes aegypti. The egg hatchability, larval mortality, pupal mortality and adult emergence of Aedes aegypti was observed separately in control and different concentration of the seed and leaf extracts of experimental plant, and in both the victims recovered uneventfully.

\section{RESULTS}

\subsection{Effect of partially purified plant extracts on the egg hatchability of Aedes aegypti}

Eggs of Aedes aegypti were treated with the partially purified plants extract for $24 \mathrm{hrs}$. The order of LC50(ppm) concentrationswere 50.46, 38.49, $25.74,58.94,39.83,72.27,72.65,88.46,82.53$ and 
82.73 ppm in the plantRicinus communis (Table 1, Fig 1).

\subsection{Effect of partially purified plant extracts on the I instar larvae of Aedes aegypti}

The first instar larvae of Aedes aegypti were exposed to plant extracts and the LC50 (ppm) values noticed were $4.57,3.38,4.15,7.86,7.35,18.68$,

22.38, 16.18, 19.66 and $24.17 \mathrm{ppm}$.The effect of plant extract were found to be in the order of Ricinus communis (Table 1,Fig 1).

\subsection{Effect of partially purified plant extracts on the II instar larvae of Aedes aegypti}

The second instar larvae of Aedes aegypti were exposed to plant extracts and the LC50(ppm) values noticed were $7.25,7.24,4.93,23.84,15.54,31.19$, 26.73, 24.24, 26.16 and $31.54 \mathrm{ppm}$.The effect of plant extract were found to be in the Ricinus communis (Table 1, Fig. 1)

\subsection{Effect of partially purified plant extracts on} the III instar larvae of Aedes aegypti

The third instar larvae of Aedes aegypti were exposed to plant extracts and the LC50(ppm) values noticed were $7.93,10.21,10.21,32.37,23.76,35.18$, 36.87, 29.16, 38.46 and $47.28 \mathrm{ppm}$.The effect of plant extract were found to be in theRicinus communis (Table 1, Fig 1).

\subsection{Effect of partially purified plant extracts on the IV} instar larvae of Aedes aegypti

The fourth instar larvae of Aedes aegypti were exposed to plant extracts and the LC50(ppm) values noticed were 25.26, 18.28, 23.22, 32.43, 41.09, 47.05, $48.35,43.64,43.64$ and $61.47 \mathrm{ppm}$.The effect of plant extract were found to be in theRicinus communis (Table 1, Fig 1).

\subsection{Effect of partially purified plant extracts on the pupae of Aedes aegypti}

When the pupae of Aedes aegypti were exposed to plant extracts, the order of the LC $50(\mathrm{ppm})$ values noticed were 29.18, 29.18, 48.17, 53.59, 51.45, 82.43, 78.94, 83.56, 83.56 and $82.25 \mathrm{ppm}$ in the plant Ricinus communis (Table 1, Fig 1).

\section{DISCUSSION}

In the present study efficacy of partially purified plant extracts on the life cycle (egg, larvae and pupa) of Aedes aegypti and biochemical parameters such as $\beta-\mathrm{N}$ acetylglucosaminidase,phosphatases and phenoloxidases (embryonic egg and IV Instar larva) were observed. The plant extracts contain active principles that the protein content of the larvae, pupae and ovary of Aedes aegypti were recorded. $\beta$ $\mathrm{N}$-acetylglucosaminidase and phosphatases are involved in moulting and the enzyme phenoloxidase may be involved in the synthesis of cuticle during embryogenesis. Preliminary phytochemicals of plant extracts were observed and GC-MS of the plant.

Botanical insecticides have been used for centuries for crop protection. Only with the development of synthetic insecticides in the mid 1900's did their use drop as more effective products took their place. Within a relatively short time, problems arise with the synthetic products.Environmental contamination, poisoning of non-target species and resistance. This led many to reconsider botanical formulations as natural alternatives because they are less toxic (Scott and Kaushik, 2004).Plants with and established record for culinary or medicinal use that therefore offer a safer starting material have been evaluated in terms of their potential application as mosquitoes .

For the current study the plant compounds were considered not as leads for synthetic insecticides, but for extract based formulations that combine all the co-occurring secondary plant compounds.Keeping the above said factors, ten medicinal plants were screened plant biopesticidal activity.Eggs, I, II, III, IV instar larvae and pupae were treated with the plant of medicinal value of Ricinus communis.

Unhatched eggs, mortality of larvae and pupae were recorded at 24 hours intervals. Dead larvae,pupae,partially emerged or deformed adults were regularly removed and counted. Live pupae were collected and observed till emergence. The inhibition of these plant compounds against eggs, I, II, III, IV instar larvae and pupae were estimated on the corresponding mortality values and LC50 values were calculated. Morphogenetic abnormalities were studied from the dead larvae,pupae, larval pupalintermediates and partly enclosed adults.

In the present study the morphogenetic abnormalities noted in the life cycle of the treated Aedes aegypti includes extension of developmental period. Enlarged pupae, partly exuviated adults with part of abdomen still in pupal case and attachment of pupal case by legs, deformities on the abdominal wings, wing deformities consisted of twisting unevenness, incomplete development and disorientation of fore and hind wings, deformed adults failed to detach themselves from their pupal cases. 
After the introduction of larvae in the test compound of different concentration,the larvae showed mortality and the surviving larvae in the treatment were seen resting in $S$ or $U$ shaped postures and stretching frequently.This behavior was not noticed in the control.Such $U$ shaped postures and frequent stretching has been described previously (Mwangi and Rembold, 1988) as characteristic of mosquito larvae reared in water treated with Melia volkensii fruit extracts.
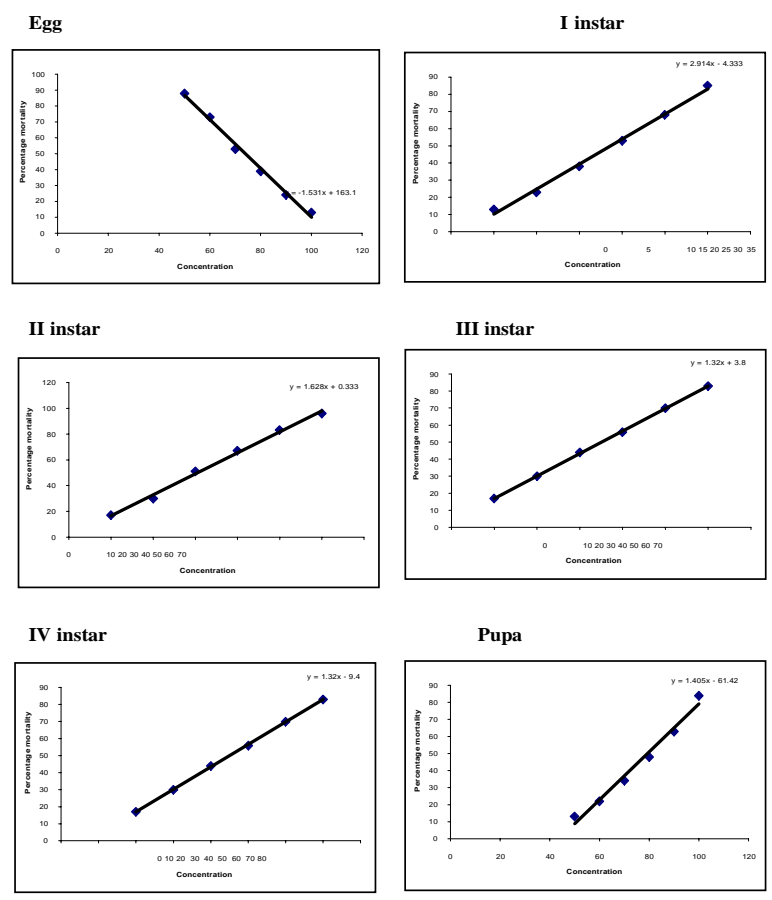

Fig. 1. Probit regression line for response of Aedes aegypti to Ricinus communis partially purified leaf extract in laboratory test

Table 1. $\mathrm{LC}_{50}(\mathrm{ppm})$ of the partially purified leaf extract of Ricinus communis on the different stages of Aedes aegypti.

\begin{tabular}{|c|c|c|c|c|c|c|c|c|}
\hline \multirow{2}{*}{$\stackrel{\rightleftarrows}{\frac{\pi}{2}}$} & \multirow[t]{2}{*}{ Stages } & \multicolumn{3}{|c|}{$\begin{array}{l}\text { LC }_{50} \text { Fiducial } \\
\text { (pplimit(ppm) }\end{array}$} & \multirow[t]{2}{*}{$\mathbf{X}^{2}$} & \multirow[t]{2}{*}{$\overline{\mathbf{x}}$} & \multirow[t]{2}{*}{ SD } & \multirow[t]{2}{*}{ SE } \\
\hline & & m) & $\begin{array}{l}\text { Upp } \\
\text { er }\end{array}$ & $\begin{array}{l}\text { Low } \\
\text { er }\end{array}$ & & & & \\
\hline \multirow{12}{*}{ 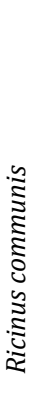 } & Egg & 72.2 & 75.6 & 69.5 & 2.2 & 55. & 26.2 & 2.6 \\
\hline & & 7 & 0 & 4 & 6 & 4 & 4 & 0 \\
\hline & I Instar & 18.6 & 20.4 & 16.7 & 1.1 & 39. & 24.9 & 2.0 \\
\hline & & 8 & 6 & 3 & 7 & 0 & 9 & 9 \\
\hline & II Instar & 31.1 & 34.3 & 28.4 & 2.5 & 49. & 27.8 & 2.2 \\
\hline & & 9 & 7 & 9 & 4 & 6 & 7 & 3 \\
\hline & III Instar & 35.1 & 38.3 & 32.1 & 1.9 & 43. & 22.5 & 0.4 \\
\hline & & 8 & 9 & 6 & 2 & 4 & 4 & 4 \\
\hline & IV Instar & 47.0 & 50.3 & 44.2 & 1.9 & 43. & 22.5 & 0.4 \\
\hline & & 5 & 3 & 8 & 1 & 4 & 4 & 4 \\
\hline & Pupa & 82.4 & 85.2 & 79.5 & 1.4 & 36. & 24.2 & 3.9 \\
\hline & & 3 & 9 & 4 & 5 & 0 & 2 & 9 \\
\hline
\end{tabular}

\section{REFERENCES}

Cheng, S.S., H.T. Chang, S.T. Chang, K.H. Tsai and W.J. Chen, (2003). Bioactivity of selected plant essential oils against the yellow fever mosquito Aedes aegypti larvae. Bioresource Technol. 89: 99-102.

Cheng, S.S., J.Y. Liu, K.H. Tsai, W.J. Chen and S.T. Chang, (2004). Chemical composition and mosquito larvicidal activity of essential oils from leaves of different Cinnamomum osmophloeum provenances. J. Agric Food Chem. 52: 4395-4400.

Rembold, F.L. and R.W. Mwang, (1989). Compounds from/ MeKa volkensi and their growth inhibitory effect on Aedes aegypti larvae. Proc second symposium of host regulated developmental mechanisms in vector arthropods at Vero Beach. Florida. 3-8.

Sagar, S.K. and S.S. Sehgal, (1996). Effects of aqueous extract of deoiled neem (Azadirachta Indica A. juss) seed kernel and karanja (Pongamia Glabra vent) seed kernel against Culex quinquefasciatus. J. Commun. Dis. 28: 260-269.

Shahi, M., A.A. Hanafi-Bojd, M. Iranshahi, H. Vatandoost and M.Y. Hanafi-Bojd, (2010). Larvicidal efficacy of latex and extract of Calotropis procera (Gentianales: Asclepidaceae) against Culex quinquefasciatus and Anopheles staphensi (Dipptera: Culicidae). J. Vector Borne Dis. 47: 185-188

Singh, N., A.K. Mishra and A. Saxena, (1996). Use of neem cream as a mosquito repellent in tribal areas of central India. Indian J. Malariol. 33: 99102.

Singh, R.K., R.C. Dhiman and S.P. Singh, (2003). Laboratory studies on the predatory potential of dragonfly nymphs on mosquito larvae. Common. Dis. 35: 96-101.

Singh, R.P. (1984). Effects of water extract of deoiled neem kernel on second instar larvae of Culex fatigans Wiedemann. Neem Newsletter. 1: 16. 